\title{
Effect of interleukin-17 on receptor-interacting protein 4 expression and keratinocyte proliferation
}

\author{
KUN JIA ${ }^{1}$, YAN ZHANG ${ }^{1}$, WEIYUAN MA ${ }^{2}$, XIAOFENG ZHANG ${ }^{1}$ and QING SUN ${ }^{2}$ \\ ${ }^{1}$ Shandong University School of Medicine; ${ }^{2}$ Department of Dermatology, Qilu Hospital, \\ Shangdong University, Jinan, Shandong 250012, P.R. China
}

Received July 16, 2014; Accepted April 28, 2015

DOI: $10.3892 /$ etm.2015.2478

\begin{abstract}
The aim of the present study was to investigate the effect of receptor-interacting protein 4 (RIP4) on keratinocyte proliferation and its role in the pathogenesis of psoriasis vulgaris. The expression of RIP4 and Ki-67 in fixed sections from 30 patients with psoriasis vulgaris and 30 gender- and age-matched healthy controls was detected by two-step immunohistochemistry, prior to the correlation being examined with Pearson's analysis. Reverse transcription-semi-quantitative polymerase chain reaction and western blot analyses were carried out to detect the mRNA and protein expression of RIP4 in an immortalized human keratinocyte line, HaCaT, stimulated by different concentrations of interleukin-17 (IL-17), in order to analyze the change in RIP4 expression following IL-17 stimulation. The cell proliferation rate was measured using the cell counting kit- 8 assay simultaneously. RIP4 was mainly present in the cytoplasm of the keratinocytes. Compared with its expression in the healthy control skin, RIP4 exhibited a significant upregulation in the psoriatic lesions $(\mathrm{P}<0.05)$. Pearson's correlation analysis revealed a positive correlation between the expression level of RIP4 and the proliferation index. Both RIP4 mRNA and protein levels were significantly increased following IL-17 stimulation. Exposure to IL-17 additionally increased the proliferation rate of the HaCaT cells. In conclusion, RIP4 may play a role in the pathogenesis of psoriasis vulgaris as a potential target of IL-17.
\end{abstract}

\section{Introduction}

Psoriasis vulgaris is a common chronic inflammatory skin disease marked by the hyperproliferation of keratinocytes and infiltration of inflammatory cells. Although its pathogenesis has not been not fully elucidated, the condition is generally

Correspondence to: Dr Qing Sun, Department of Dermatology, Qilu Hospital, Shandong University, 107 West Wenhua Road, Jinan, Shandong 250012, P.R. China

E-mail: qlderlab@163.com

Key words: receptor-interacting protein 4, interleukin-17, psoriasis vulgaris, keratinocytes, proliferation recognized as a polygenic disease resulting from a dysregulated interplay between hereditary and environmental factors, which gives rise to the hyperproliferative epidermis and altered differentiation through an immune-mediated common pathway. Immune dysfunction plays a vital role in the pathogenic progression of psoriasis vulgaris $(1,2)$. $T$ cells comprise the majority of the immune response cells in the lesions. A large number of T-helper (Th) cells can be found infiltrating the inflammatory reaction area, and one pathway that is important in psoriasis is the role of the Th17/interleukin-17 (IL-17) dysregulation (3-5). Th17 cells are a T-cell subset distinct from both Th1 and Th2 cells. IL-17, which is predominantly produced by Th17 cells, has been implicated in the pathogenesis of psoriasis vulgaris (6-8).

Receptor-interacting protein 4 (RIP4), as a novel member of the RIP kinase family, activates mitogen-activated protein kinases (MAPKs) and nuclear factor $\kappa$-light-chain-enhancer of activated $\mathrm{B}$ cells $(\mathrm{NF}-\kappa \mathrm{B})$ during the proliferation, differentiation, apoptosis, inflammation and immune response of keratinocytes $(9,10)$. The present study aims to investigate the role of RIP4 in psoriasis vulgaris through the use of immunohistochemistry to examine the RIP4 expression in the condition, and by detecting the mRNA and protein levels of RIP4 in a human immortal keratinocyte cell line, $\mathrm{HaCaT}$, following stimulation of the cells with graded IL-17 concentrations.

\section{Materials and methods}

Patients and skin biopsies. Thirty psoriatic biopsies were obtained from 15 male and 15 female patients (mean age, $27.96 \pm 8.64$ years; range, $19-52$ years) who had been diagnosed with psoriasis vulgaris in Qilu Hospital (Jinan, China) and who had not received any local or systemic treatment since the latest attack of the disease. Non-lesion biopsies, acquired from 30 age- and gender-matched healthy individuals, served as controls. All participants were negative for systemic disease and gave their informed consent prior to biopsy. The study received approval from the Ethics Committee of Qilu Hospital (no. QL2009019). The specimens were fixed in 10\% neutral formaldehyde immediately subsequent to their collection.

Immunohistochemistry. Paraffin-embedded $10 \%$ neutral formaldehyde-fixed tissues were sectioned continuously at $3 \mu \mathrm{m}$, deparaffinized with xylene and rehydrated in a graded alcohol series. For antigen retrieval, the deparaffinized 
sections in $1 \mathrm{mM}$ EDTA buffer $(\mathrm{pH}=9.0)$ were heated at $120^{\circ} \mathrm{C}$ for $5 \mathrm{~min}$ and cooled slowly to room temperature. In order to prevent nonspecific background staining, the slices were blocked with goat serum following the quenching of endogenous peroxidase activity with $3 \% \mathrm{H}_{2} \mathrm{O}_{2}$ solution. Polyclonal rabbit anti-human RIP4 antibody (cat. no. ZA-0502; Santa Cruz Biotechnology, Inc., Santa Cruz, CA, USA), at a dilution of 1:100, and a ready-to-use anti-Ki-67 monoclonal antibody (ZSGB-Bio, Beijing, China) were applied to the sections at $37^{\circ} \mathrm{C}$ for $1 \mathrm{~h}$, prior to the use of the PV-6000 polymer detection system (ZSGB-Bio) according to the manufacturer's instructions. 3,3'-Diaminobenzidine (ZSGB-Bio) was applied to visualize the antibody under a microscope, following which the sections were counterstained with Harris's hematoxylin. The slices were mounted with neural gum and images were captured using the ToupCam microscope camera system (Hangzhou ToupTek Photonics Co., Ltd., Hangzhou, China).

Assessment of immunohistochemistry. Cells were considered to be RIP4- and Ki-67-positive when yellow to brown granules appeared in the cytoplasm or nucleus of the keratinocytes, respectively. The proliferation index was calculated as a percentage by dividing the number of Ki-67-positive cells by the total number of epidermal cells (basal and suprabasal). Five high-power fields (x400 magnification) were counted in each sample.

Cell culture. Immortalized human keratinocyte HaCaT cells were obtained commercially from the American Type Culture Collection (Manassas, VA, USA) and frozen in our lab. The $\mathrm{HaCaT}$ cells were thawed using a routine method and grown in Dulbecco's modified Eagle's medium (Gibco-BRL, Grand Island, NY, USA) supplemented with $10 \%$ (vol/vol) fetal bovine serum, $100 \mathrm{U} / \mathrm{ml}$ penicillin and $100 \mu \mathrm{g} / \mathrm{ml}$ streptomycin in a humidified atmosphere containing $5 \% \mathrm{CO}_{2}$ at $37^{\circ} \mathrm{C}$. For the subcultures, the cells were disaggregated with $0.25 \%$ trypsin/0.02\% EDTA (1:1) solution and split at a ratio of 1:2 every 2 to 3 days, prior to inoculation into 12-well plates at a concentration of $1 \times 10^{5}$ cells $/ \mathrm{ml}$. HaCaT cells at 60-70\% confluence were stimulated with IL-17 (50, 70 and 90 ng/ml; ProSpec-Tany TechnoGene, Ltd., Rehovot, Israel) for RNA isolation or western blotting. The control group cells were processed in parallel using Dulbecco's modified Eagle's medium without IL-17.

Cell counting kit-8 (CCK-8) assay. The CCK-8 assay (Dojindo Molecular Technologies, Inc., Kumamoto, Japan) was adopted to evaluate cell viability. Cells $\left(5 \times 10^{3} / \mathrm{ml}\right)$ in the logarithmic phase were inoculated in 96-well plates with $100 \mu 1$ normal growth medium containing $10 \%$ fetal calf serum. After $24 \mathrm{~h}$, the medium was changed to serum-free medium. When the cell reached $\sim 70 \%$ confluence, various doses of IL-17 (50, 70 and $90 \mathrm{ng} / \mathrm{ml}$ ) in serum-free medium were added. A total of $10 \mu \mathrm{l} \mathrm{CCK}-8$ was added to each well after 12, 24, 36 and $48 \mathrm{~h}$ incubation with IL-17, prior to further incubation for $2 \mathrm{~h}$ at $37^{\circ} \mathrm{C}$. A microplate reader (Varioskan ${ }^{\mathrm{TM}}$ Flash; Thermo Fisher Scientific, Inc., Waltham, MA, USA) was applied to measure the optical density (OD) of each well at $450 \mathrm{~nm}$. The cell viability (percentage of control) is expressed as the percentage of $\left(\mathrm{OD}_{\text {test }}-\mathrm{OD}_{\text {blank }}\right) /\left(\mathrm{OD}_{\text {control }}-\mathrm{OD}_{\text {blank }}\right)$.
Table I. Primer sequences.

\begin{tabular}{ll}
\hline Gene & \multicolumn{1}{c}{ Primer sequence } \\
\hline RIP4 & $\begin{array}{l}\text { Upstream: 5'-TGTTAGGTGATTTGGGATAGG-3' } \\
\text { Downstream: 5'-AAAGGCACAATGAGGCATA-3' }\end{array}$ \\
\multirow{3}{*}{-actin } & $\begin{array}{l}\text { Upstream: 5'-CATTTGCTGCATGGGTTA-3' } \\
\text { Downstream: 5'-TCCTACGGCTTGGACTTT-3' }\end{array}$
\end{tabular}

RIP4, receptor-interacting protein 4.

Reverse-transcription (RT)-semiquantitative polymerase chain reaction $(P C R)$. Total RNA was isolated from the cells $48 \mathrm{~h}$ after stimulation with IL-17 using RNAiso Plus [Takara Biotechnology (Dalian) Co., Ltd., Dalian, China] in accordance with the manufacturer's instructions and quantified spectrophotometrically at $260 \mathrm{~nm}$. A total of $1 \mu \mathrm{g}$ RNA was converted in cDNA using oligo (dT) primers and then amplified with a Power RT kit (BioTeke, Beijing, China). The PCR was performed in a total reaction volume of $50 \mu \mathrm{l}$ : $25 \mu \mathrm{l} 2 \mathrm{X}$ PCR Mix (BioTeke), $2.5 \mu \mathrm{l}$ forward and reverse primer, $10 \mu \mathrm{l}$ templates and $10 \mu \mathrm{l}$ sterile $\mathrm{H}_{2} \mathrm{O}$. The mixture underwent predenaturation for $5 \mathrm{~min}$ at $95^{\circ} \mathrm{C}$ and was then subjected to 35 cycles of $95^{\circ} \mathrm{C}$ for $1 \mathrm{~min}, 60^{\circ} \mathrm{C}$ for $45 \mathrm{sec}$ and $72^{\circ} \mathrm{C}$ for $45 \mathrm{sec}$, and finally $72^{\circ} \mathrm{C}$ for $10 \mathrm{~min}$. The specific primer sets (Table I) were commercially synthesized by Shanghai Sangon Biotechnology Co., Ltd. (Shanghai, China). The amplified DNA fragments were analyzed using $2 \%$ agarose gel electrophoresis. The expected fragment length was $384 \mathrm{bp}$ for RIP4 and 447 bp for $\beta$-actin. Ethidium bromide-stained PCR products were visualized using an ultraviolet transilluminator (Tanon $^{\mathrm{TM}}$ 2500R; Tanon Science \& Technology Co., Ltd., Shanghai, China), and the gray value of the DNA bands was simultaneously determined using an automatic image analyzer with the following formula: Relative gray value $=$ RIP4/ $\beta$-actin .

Western blot analysis. HaCaT cells were dissolved in lysis buffer (cell lysis buffer for western and immunoprecipitation; Beyotime Institute of Biotechnology, Haimen, China) containing $1 \mathrm{mM}$ phenylmethanesulfonyl fluoride (Beyotime Institute of Biotechnology). The mixture was centrifuged for $5 \mathrm{~min}$ at $12,000 \mathrm{x} \mathrm{g}$ and the bicinchoninic acid (BCA) assay method (Enhanced BCA Protein Assay kit; Beyotime Institute of Biotechnology) was utilized to quantify the total protein in the supernatant. A total of $30 \mu \mathrm{g}$ protein from the cell lysate was solubilized in loading buffer and subjected to sodium dodecyl sulfate-polyacrylamide gel electrophoresis on a $10 \%$ acrylamide gel, prior to being electrotransferred onto polyvinylidene difluoride (PVDF) membranes. Following blocking with $5 \%$ skimmed milk at room temperature for $1 \mathrm{~h}$, the PVDF membranes were incubated overnight at $4^{\circ} \mathrm{C}$ with polyclonal rabbit anti-human RIP4 antibody at a dilution of 1:400 (sc-83320; Beijing Biosynthesis Biotechnology Co., Ltd., Beijing, China) or with mouse anti-human anti- $\beta$-actin antibody (Santa Cruz Biotechnology, Inc.) at a dilution of 1:1,000. The membranes were then thoroughly washed with $0.01 \mathrm{M}$ Tris-buffered saline containing $0.1 \%$ Tween 20 and incubated with horseradish peroxidase-labeled goat-anti-rabbit or goat-anti-mouse immunoglobulin G (heavy- and light-chain) antibody (EarthOx Life 

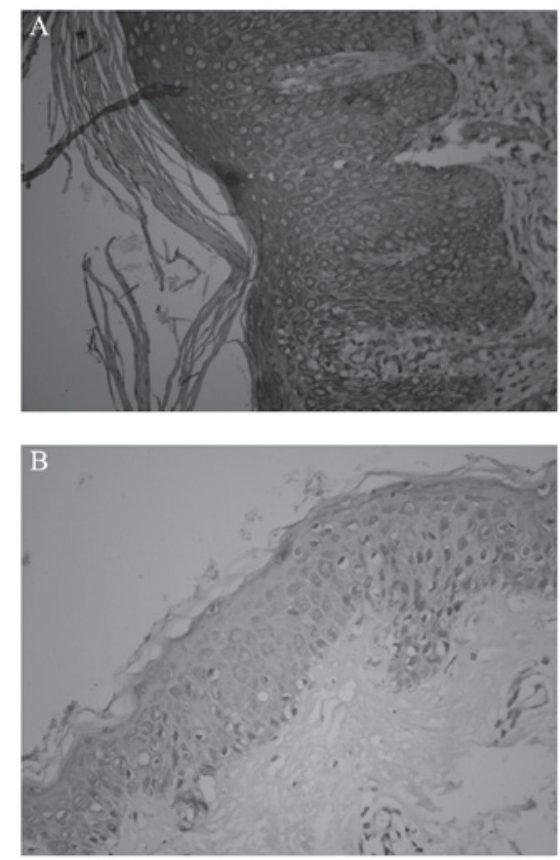

Figure 1. Receptor-interacting protein 4 expression in (A) psoriatic lesions and (B) healthy controls. Magnification, x200.

Sciences, Millbrae, CA, USA) at a dilution of 1:1,000 at room temperature for $1 \mathrm{~h}$. A standard enhanced chemiluminescence (Shanghai Sangon Biotechnology Co., Ltd.) reaction was performed according to the manufacturer's instructions, and densitometric analysis of the band intensity was conducted using a chemiluminescence imaging analysis system (Tanon 5000; Tanon Science \& Technology Co., Ltd.). Relative expression was measured by dividing the gray value of RIP4 by that of $\beta$-actin. High gray values represented low expression.

Statistical analysis. All statistical analysis was carried out using the SPSS 17.0 software package (SPSS, Inc., Chicago, IL, USA), and data are presented as the mean \pm standard error of the mean. The Student's t-test was performed for the comparison of the results, and the correlation between RIP4 expression and the proliferation index was examined using Pearson's correlation analysis.

\section{Results}

RIP4 is mainly expressed in the cytosol and nuclei of keratinocytes and shows an evident upregulation in psoriatic lesions. In healthy skin, RIP4 was found predominantly in the basal layer of the epidermis; by contrast, the expression in the psoriatic lesions was concentrated in the basal and spinous layers, in the cytoplasm and nuclei of the cells. Analysis of the OD showed the mean OD (MOD) of RIP4 in the healthy controls to be $0.11 \pm 0.03$, compared with $0.30 \pm 0.05$ in the psoriasis vulgaris lesions. This revealed a statistically significant upregulation in the psoriatic lesions $(\mathrm{t}=7.71, \mathrm{P}<0.01)$ (Figs. 1 and 2$)$.

RIP4 expression in psoriatic skin is associated with keratinocyte proliferation. In the healthy control skin, Ki-67-positive cells only appeared sporadically in the basal layer. By contrast, $\mathrm{Ki}$-67-positive cells in the psoriatic specimens were distrib-

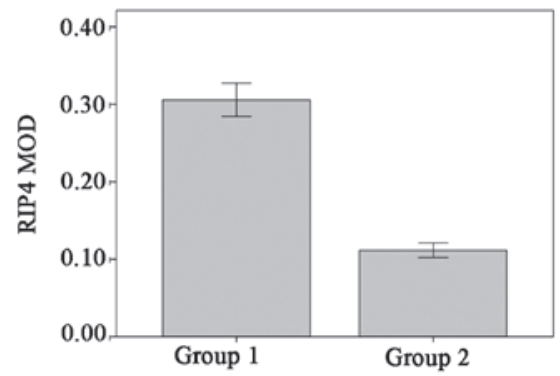

Figure 2. Difference in RIP4 expression between the psoriatic lesions (group 1) and healthy controls (group 2) $(\mathrm{t}=7.71, \mathrm{P}<0.01)$. Data are presented as the mean \pm standard error of the mean. RIP4, receptor-interacting protein 4 ; MOD, mean optical density.

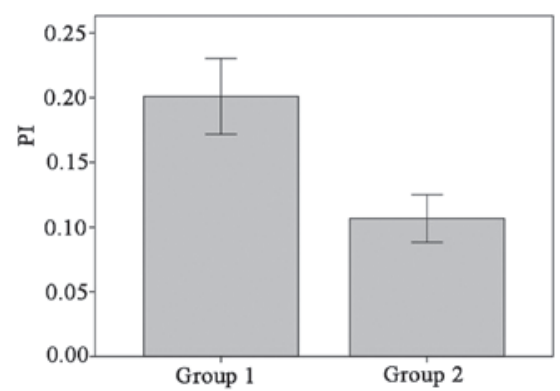

Figure 3. Difference in Ki-67 expression (used to calculate PI) between the psoriatic lesions (group 1) and healthy controls (group 2) $(\mathrm{t}=4.535, \mathrm{P}<0.05)$. Data are presented as the mean \pm standard error of the mean. PI, proliferation index.

uted in not only the basal layer but also the middle and lower parts of the rete ridges of the lesional epidermis. The proliferation index of the psoriatic lesions was $20.01 \pm 7.90 \%$, while that of the healthy control skin was $10.67 \pm 4.92 \%$; this suggested that the $\mathrm{Ki}-67$ expression in the psoriatic lesions was significantly higher than that in the healthy control skin $(\mathrm{t}=4.535$, $\mathrm{P}<0.05$ ) (Fig 3). In addition, Pearson's correlation analysis demonstrated a significantly positive correlation between the RIP4 MOD and the proliferation index, which indicated that a high level of RIP4 expression led to the active proliferation of keratinocytes in the epidermis $(\mathrm{r}=0.692, \mathrm{P}<0.01)$ (Fig. 4).

Promotion of HaCaT cell proliferation and significant upregulation of RIP4 following IL-17 stimulation. To further investigate the role of RIP4 in psoriasis, an immortalized line of human epidermal keratinocytes, $\mathrm{HaCaT}$, which has been extensively adopted as an in vitro model for studies of psoriasis, was utilized. The CCK-8 assay performed 12, 24 , 36 and $48 \mathrm{~h}$ after culture in medium containing IL-17 revealed that IL-17 at concentrations of between 50 and $90 \mathrm{ng} / \mathrm{ml}$ had a marked stimulatory effect on $\mathrm{HaCaT}$ cell proliferation, acting in a dose- and time-dependent manner. Increased concentrations of IL-17 resulted in higher proliferation rates (Fig. 5).

RT-semi-quantitative PCR and western blot analyses were applied to study the change in RIP4 mRNA and protein expression, respectively, following exposure to IL-17. The RIP4 mRNA and protein were isolated $48 \mathrm{~h}$ after the stimulation of the cells with 50,70 and $90 \mathrm{ng} / \mathrm{ml} \mathrm{IL-17}$ and subjected to RT-semi-quantitative PCR or western blot analyses with 


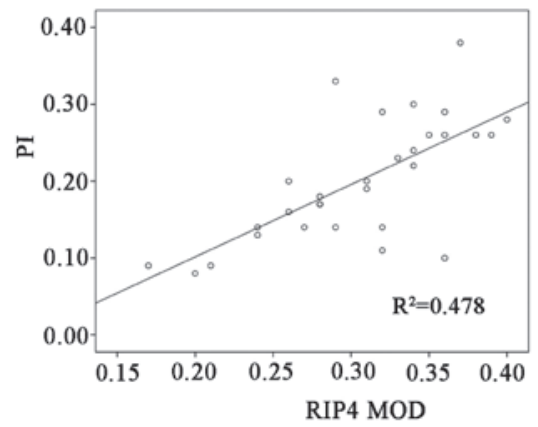

Figure 4. Correlation of RIP4 expression and the PI $(r=0.69, \mathrm{P}<0.01)$. RIP4, receptor-interacting protein 4 ; MOD, mean optical density; PI, proliferation index.

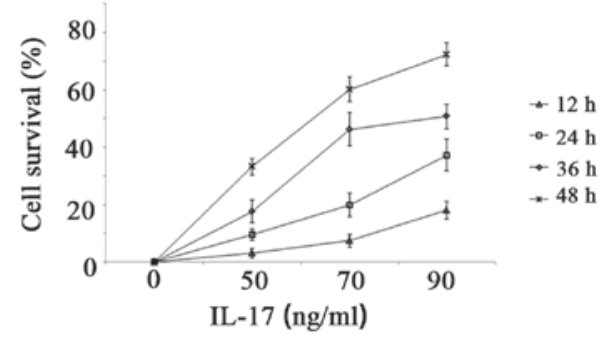

Figure 5. Proliferation index of HaCaT cells following stimulation with different concentration of IL-17. Data are presented as the mean \pm standard error of the mean. IL-17, interleukin-17.

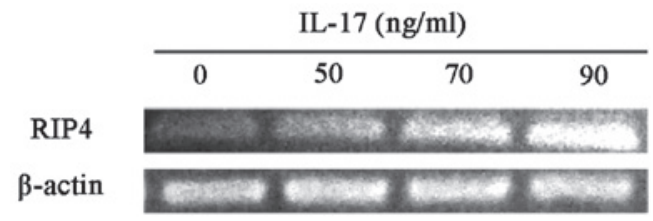

Figure 6. Reverse transcription-semi-quantitative polymerase chain reaction analysis of RIP4 mRNA following IL-17 stimulation. RIP4, receptor-interacting protein 4; IL-17, interleukin-17.

$\beta$-actin as a housekeeping control. Compared with the control group, it was found that IL-17 dose-dependently induced a significant upregulation of RIP4 expression at both the mRNA $\left(\mathrm{t}_{50}=5.091, \mathrm{P}_{50}=0.036 ; \mathrm{t}_{70}=7.022, \mathrm{P}_{70}=0.020 ; \mathrm{t}_{90}=18.621\right.$, $\left.\mathrm{P}_{90}=0.003\right)$ and protein $\left(\mathrm{t}_{50}=9.076, \mathrm{P}_{50}=0.012 ; \mathrm{t}_{70}=6.600\right.$, $\mathrm{P}_{70}=0.022 ; \mathrm{t}_{90}=6.650, \mathrm{P}_{90}=0.022$ ) levels (Figs. 6-9).

\section{Discussion}

Psoriasis vulgaris is a common chronic skin disease in which a thicker epidermis, excessive keratinocyte proliferation and parakeratosis, accompanied by extensive inflammatory cell infiltration, can be observed (9). It is generally believed that the abnormal keratinocyte proliferation and differentiation occurs as a result of $\mathrm{T}$ cells recognizing a previously unidentified self-antigen, giving rise to an immune response $(10,11)$. To date, considerable evidence has revealed the aggregation of Th17 cells and the upregulation of IL-17 and IL-17 receptor (IL-17R) in psoriatic lesions $(12,13)$. Furthermore, the application of agents that act to deplete the number of $\mathrm{T}$ cells in

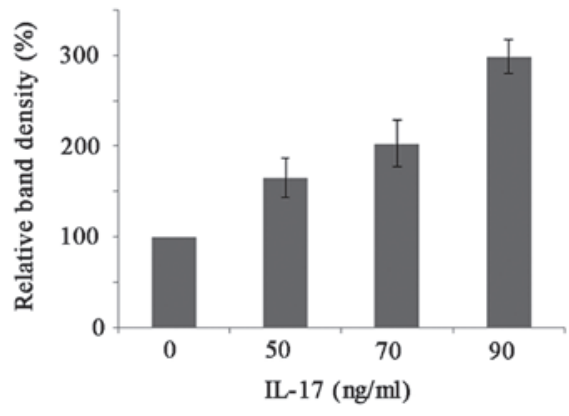

Figure 7. Difference in receptor-interacting protein $4 \mathrm{mRNA}$ levels following IL-17 stimulation: $\mathrm{t}_{50}=5.091, \mathrm{P}_{50}=0.036 ; \mathrm{t}_{70}=7.022, \mathrm{P}_{70}=0.020 ; \mathrm{t}_{90}=18.621$, $\mathrm{P}_{90}=0.003$. Data are presented as the mean \pm standard error of the mean. IL-17, interleukin-17.

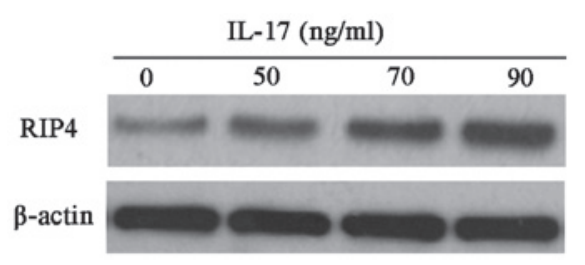

Figure 8. Western blot analysis of RIP4 protein following IL-17 stimulation RIP4, receptor-interacting protein 4; IL-17, interleukin-17.

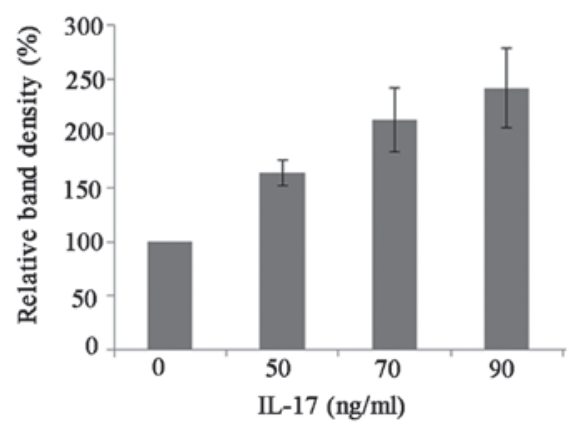

Figure 9. Difference in receptor-interacting protein 4 protein levels following IL-17 stimulation: $\mathrm{t}_{50}=9.076, \mathrm{P}_{50}=0.012 ; \mathrm{t}_{70}=6.600, \mathrm{P}_{70}=0.022 ; \mathrm{t}_{90}=6.650$, $\mathrm{P}_{90}=0.022$. Data are presented as the mean \pm standard error of the mean. IL-17, interleukin-17.

skin lesions and the administration of monoclonal anti-IL-17 antibodies have been shown to lead to the clinical and histopathological remission of psoriasis vulgaris, thereby providing direct evidence that the IL-17-induced changes in the proliferation and differentiation of keratinocytes play a role in the pathogenesis of psoriasis vulgaris (12-16).

The RIP kinase family comprises a group of specific serine/threonine kinases that are crucial mediators of multiple signal transduction processes, which lead to the activation of NF- $\kappa B$ and MAPKs (17). RIP4, a novel member of the RIP kinase family, was initially identified as a kinase that interacts with an isoform of the protein kinase C (PKC) family, PKC $\delta$. RIP4 shares a homologous N-terminal kinase domain with other RIP kinases ( 40\% identity) and is characterized by a C-terminal region harboring 11 ankyrin repeats. RIP4 is widespread in various tissues and cells throughout the body, including the liver, kidney, brain, heart, lung, skeletal muscles and skin. Overexpression of RIP4 in 293T cells results in the 
activation of NF- $\mathrm{kB}$ and the c-Jun N-terminal kinase (JNK) signaling pathway in a dose-dependent manner (17). Previous studies (18-20) have demonstrated that RIP4 is required during epidermal differentiation and cell proliferation in mice, based on the fact that epidermal dysplasia was observed in RIP4-deficient mice. The outermost cornified layers were absent in RIP4 ${ }^{-1}$ skin and were replaced by a thick layer of flattened, parakeratotic cells with abnormal expression of the proteins that characteristically mark the specific epidermal layers. Furthermore, marked hyperplasia of the spinous and granular layers was observed, indicating that RIP4 is a crucial factor in the embryonic development of the epidermis (18). The strong downregulation of RIP4 following skin injury has indicated it to be an important regulator of re-epithelialization following injury (21). In addition, K14-RIP4 mice are sensitive to cutaneous inflammation with markedly overexpressed RIP4 (19). Thus, we suggest that RIP4 could be a key element in the regulation of keratinocyte proliferation and differentiation and the inflammatory response.

RIP4 activates NF- $\kappa B$ by inducing inhibitor of $\kappa B$ phosphorylation, which requires an active kinase domain. Similar to NF- $\kappa$ B activation, RIP4-mediated JNK signal initiation depends on the presence of the kinase domain $(22,23)$. Elevated levels of NF- $\mathrm{KB}$ and JNK can be observed in psoriatic lesions, and drugs aiming to inactivate NF- $\kappa \mathrm{B}$ and block the JNK signaling pathway appear to be of satisfactory efficacy (24-26). It is speculated that the RIP4-mediated overactivation of $\mathrm{NF}-\kappa \mathrm{B}$ and JNK may contribute to such pathological processes as active keratinocyte proliferation.

IL-17 is one of the most important inflammatory factors in psoriasis. In the present study, the elevated proliferation rate of $\mathrm{HaCaT}$ cells and the higher levels of RIP4 expression were shown to be enhanced by stimulation with an increasing concentration of IL-17. Furthermore, the RIP4 protein level, which was shown to be positively correlated with the proliferation index, was notably upregulated in the psoriatic lesions compared with the healthy control skin. It could thus be inferred that RIP4 is a possible downstream molecule of IL-17.

Interacting with IL-17R on the surface of keratinocytes, IL-17 produces proinflammatory and proliferation-regulatory effects by means of activating NF- $\mathrm{KB}$ and the JNK signaling pathway, in which RIP4 is directly involved (27). In combination, these data suggest that epidermal hyperproliferation and prosoplasia in psoriasis vulgaris could be the result of overexpressed RIP4, induced by the activation of NF- $\mathrm{KB}$ and the JNK signaling pathway by elevated IL-17. In conclusion, IL-17, as an inflammatory factor, could lead to the upregulation of RIP4 in keratinocytes, and the IL-17/RIP4 axis may play a role in the epidermal hyperplasia of psoriasis vulgaris.

\section{Acknowledgements}

This study was supported by the 2010 National Science Foundation (grant no. 81071291).

\section{References}

1. Ariza ME, Williams MV and Wong HK: Targeting IL-17 in psoriasis: From cutaneous immunobiology to clinical application. Clin Immunol 146: 131-139, 2013.

2. Bowcock AM and Krueger JG: Getting under the skin: The immunogenetics of psoriasis. Nat Rev Immunol 5: 699-711, 2005
3. Gudjonsson JE, Johnston A, Sigmundsdottir $\mathrm{H}$ and Valdimarsson H: Immunopathogenic mechanisms in psoriasis. Clin Exp Immunol 135: 1-8, 2004.

4. Valdimarsson H, Bake BS, Jónsdótdr I and Fry L: Psoriasis: A disease of abnormal Keratinocyte proliferation induced by T lymphocytes. Immunol Today 7: 256-259, 1986.

5. Ghoreschi K, Weigert C and Röcken M: Immunopathogenesis and role of T cells in psoriasis. Clin Dermatol 25: 574-580, 2007.

6. Di Cesare A, Di Meglio P and Nestle FO: The IL-23/Th17 axis in the immunopathogenesis of psoriasis. J Invest Dermatol 129: 1339-1350, 2009.

7. Blauvelt A: T-helper 17 cells in psoriatic plaques and additional genetic links between IL-23 and psoriasis. J Invest Dermatol 128: 1064-1067, 2008.

8. Chiu HY, Cheng YP and Tsai TF: T helper type 17 in psoriasis: From basic immunology to clinical practice. Dermatol Sin 30: 136-141, 2012.

9. Meylan E and Tschopp J: The RIP kinases: Crucial integrators of cellular stress. Trends Biochem Sci 30: 151-159, 2005.

10. McKay IA and Leigh IM: Altered keratinocyte growth and differentiation in psoriasis. Clin Dermatol 13: 105-114, 1995.

11. Tschachler E: Psoriasis: The epidermal component. Clin Dermatol 25: 589-595, 2007.

12. Lowes MA, Kikuchi T, Fuentes-Duculan J, et al: Psoriasis vulgaris lesions contain discrete populations of Th1 and Th17 T cells. J Invest Dermatol 128: 1207-1211, 2008.

13. Zhang L, Yang XQ, Cheng J, et al: Increased Th17 cells are accompanied by FoxP3(+) Treg cell accumulation and correlated with psoriasis disease severity. Clin Immunol 135: 108-117, 2010.

14. Krueger JG, Fretzin S, Suárez-Fariñas M, et al: IL-17A is essential for cell activation and inflammatory gene circuits in subjects with psoriasis. J Allergy Clin Immunol 130: 145-154, 2012.

15. Leonardi C, Matheson R, Zachariae C, et al: Anti-interleukin-17 monoclonal antibody ixekizumab in chronic plaque psoriasis. New Engl J Med 366: 1190-1199, 2012.

16. Papp KA, Leonardi C, Menter A, et al: Brodalumab, an anti-interleukin-17-receptor antibody for psoriasis. New Engl J Med 366: 1181-1189, 2012.

17. Meylan E, Martinon F, Thome M, et al: RIP4 (DIK/PKK), a novel member of the RIP kinase family, activates NF-kappa B and is processed during apoptosis. EMBO Rep 3: 1201-1208, 2002.

18. Holland PM, Willis CR, Kanaly S, et al: RIP4 is an ankyrin repeat-containing kinase essential for keratinocyte differentiation. Curr Biol 12: 1424-1428,2002.

19. Rountree RB, Willis CR, Dinh H, et al: RIP4 regulates epidermal differentiation and cutaneous inflammation. J Invest Dermatol 130: 102-112, 2010.

20. Adams S and Munz B: RIP4 is a target of multiple signal transduction pathways in keratinocytes: Implications for epidermal differentiation and cutaneous wound repair. Exp Cell Res 316: 126-137, 2010.

21. Adams S, Pankow S, Werner S and Munz B: Regulation of NF-kappaB activity and keratinocyte differentiation by the RIP4 protein: Implications for cutaneous wound repair. J Invest Dermatol 127: 538-544, 2007.

22. Bell S, Degitz K, Quirling M, et al: Involvement of NF-kappaB signalling in skin physiology and disease. Cell Signal 15: 1-7, 2003.

23. Lizzul PF, Aphale A, Malaviya R, et al: Differential expression of phosphorylated NF-kappaB/RelA in normal and psoriatic epidermis and downregulation of NF-kappaB in response to treatment with etanercept. J Invest Dermatol 124: 1275-1283, 2005.

24. Gazel A, Banno T, Walsh R and Blumenberg M: Inhibition of JNK promotes differentiation of epidermal keratinocytes. J Biol Chem 281: 20530-20541, 2006.

25. Kim HR, Cho ML, Kim KW, et al: Up-regulation of IL-23p19 expression in rheumatoid arthritis synovial fibroblasts by IL-17 through PI3-kinase-, NF-kappaB- and p38 MAPK-dependent signalling pathways. Rheumatology (Oxford) 46: 57-64, 2007.

26. Awane M, Andres PG, Li DJ and Reinecker HC: NF-kappa B-inducing kinase is a common mediator of IL-17-, TNF-alpha-, and IL-1 beta-induced chemokine promoter activation in intestinal epithelial cells. J Immunol 162: 5337-5344, 1999.

27. Dong C: Diversification of T-helper-cell lineages: Finding the family root of IL-17-producing cells. Nat Rev Immunol 6: 329-333, 2006. 